\title{
DISEÑO METODOLÓGICO PARA LA SELECCIÓN DE UNA MUESTRA REPRESENTATIVA DE ESTUDIANTES UNIVERSITARIOS
}

ISSN 2219-6722

ISSNE 2222-2707

José Roberto Arrazola*, Universidad Nacional Autónoma de Honduras (UNAH), Facultad de Ciencias Económicas, Administrativas y Contables (FCEAC), Instituto de Investigaciones Económicas y Sociales (IIES), Ciudad universitaria, edificio C2, primer piso, Tel/fax: (504) 2239-1849

E-mail: jarrazola@iies-unah.org

Julio César Zavala U., Universidad Nacional Autónoma de Honduras (UNAH), Facultad de Ciencias Económicas, Administrativas y Contables (FCEAC), Instituto de Investigaciones Económicas y Sociales (IIES), Ciudad universitaria, edificio C2, primer piso, Tel/fax: (504) 2239-1849

E-mail: zavalajulio@iies-unah.org

Revista Economía y Administración (E\&A)

\section{RESUMEN}

Las instituciones de educación superior juegan un papel fundamental en la gestión, transmisión y creación del conocimiento, lo que permite formular teorías y métodos que contribuyen a la resolución de diversos problemas económicos y sociales. Los estudiantes universitarios, que son los principales demandantes del sistema de educación superior, interactúan en un ambiente heterogéneo donde convergen diferentes factores que son de interés para la comunidad científica. En la última década, muchos investigadores se han preocupado por comprender y contextualizar el entorno donde los estudiantes universitarios se encuentran, así como medir la percepción de éstos en relación a diversos aspectos políticos, económicos y sociales. Debido al tamaño considerablemente grande de la población de estudiantes universitarios, muchas veces es necesario recurrir a una muestra de éstos para analizarla y posteriormente expandir los resultados a la población en general. En el presente artículo se desarrolla un diseño metodológico para la selección de una muestra representativa de estudiantes universitarios, elaborando una conceptualización de la teoría de muestreo, así como un análisis detallado de la metodología necesaria para la estratificación, afijación, selección y cálculo de los factores de expansión de la muestra, abriendo las puertas a futuras investigaciones en centros de educación superior.

Palabras clave: muestreo, muestra, estudiantes universitarios, estratificación, factor de expansión. 


\title{
DESIGN METHODOLOGY FOR THE SELECTION OF A REPRESENTATIVE SAMPLE OF UNIVERSIDAD NACIONAL AUTÓNOMA DE HONDURAS'S STUDENTS \\ ISSN 2219-6722 \\ ISSNE 2222-2707
}

\begin{abstract}
José Roberto Arrazola*, Universidad Nacional Autónoma de Honduras (UNAH), Facultad de Ciencias Económicas, Administrativas y Contables (FCEAC), Instituto de Investigaciones Económicas y Sociales (IIES), Ciudad universitaria, edificio C2, primer piso, Tel/fax: (504) 2239-1849

E-mail: jarrazola@iies-unah.org
\end{abstract}

Julio César Zavala U., Universidad Nacional Autónoma de Honduras (UNAH), Facultad de Ciencias Económicas, Administrativas y Contables (FCEAC), Instituto de Investigaciones Económicas y Sociales (IIES), Ciudad universitaria, edificio C2, primer piso, Tel/fax: (504) 2239-1849

E-mail: zavalajulio@iies-unah.org

\begin{abstract}
Higher education institutions play a fundamental role in the management, transmission and creation of knowledge, which allows to formulate theories and methods that contribute to solving social problems. College students, who are the main demanders of the higher educational system interact in a heterogeneous environment where converging factors that are of interest to the scientific community. In the last decade, many researchers have been concerned to understand and contextualize the environment where college students are and to measure their perception in relation to different political, economic and social dimensions. Due to the considerably large size of the population of college students, it is often necessary to use a sample of these to analyze and then expand the results to the general population. This paper elaborates a methodology design for the selection of a representative sample of college students, developing a conceptualization of sampling theory and a detailed analysis of the methodology for stratification, affixation, selection and calculation of the expansion's factors of the sample, opening the door to future research in higher educations.
\end{abstract}

Keywords: Sampling, sample, university students, stratification, expansion factor.

Revista Economía y Administración (E\&A) 


\section{INTRODUCCIÓN}

La educación superior (ES), en su rol de gestora, transmisora y creadora de conocimiento, tiene la obligación de contribuir al desarrollo y mejoramiento de la calidad de vida de la sociedad en general. De esta forma, las universidades deben orientar sus esfuerzos a la formación de profesionales competentes, con amplios conocimientos (científicos y empíricos) que hagan posible crear métodos y modelos que utilicen de manera económica y sostenible los recursos para la producción, lo que permite que ante un determinado problema se pueda obtener una solución que funcione más satisfactoriamente en beneficio de la sociedad (Pérez, 2007).

La ES puede comprenderse como una cadena de suministro cuyo insumo principal son los estudiantes, los cuales ingresan a una institución de educación superior y atraviesan diferentes procesos hasta egresar como profesionales (Ortega y Eguía, 2011). Para estudiar ésta cadena de suministro de ES, es necesario comprender las características de la población de estudiantes universitarios, ya que son los actores fundamentales del proceso de educación superior, por

Revista Economía y Administración (E\&A) ello, han sido el foco de numerosas investigaciones a nivel internacional, con el objeto de conocer la percepción que éstos poseen en relación a diversos aspectos políticos (Echavarría et al., 2008; Galindo et al., 2010), económicos (Núñez et al., 2009; Castro et al., 2013; Arué, 2011) y sociales (Vásquez et al., 2009; Arana et al., 2011; González et al., 2009), así como caracterizar el contexto en que los estudiantes universitarios se encuentran (Miratía, 2010; Avendaño, 2005; Inzunsa y Jiménez, 2013).

Para realizar investigaciones cuantitativas, cuya población objetivo sean estudiantes universitarios, pueden utilizarse diferentes metodologías (exámenes, entrevistas, registros administrativos, etc.), las cuales dependen de las características de la investigación. Sin embargo, el número de estudiantes universitarios suele ser muy grande, por lo que un censo puede ser inviable por razones de tiempo, recursos o por la propia naturaleza de la investigación. La solución a éste problema puede presentarse por medio de la selección de una muestra representativa de estudiantes universitarios, a partir de la cual se realizan inferencias sobre la población.

Debido a la complejidad que requiere la selección de una muestra representativa de estudiantes universitarios, muchos investigadores optan por cambiar su diseño de investigación o peor aún, seleccionar incorrectamente una muestra y expandir los resultados obtenidos a toda la población, en este caso las conclusiones estarán 
sesgadas y no se podrá tener una medida de confiabilidad en los resultados. La forma de seleccionar una muestra representativa no es única, existen diversas metodologías que se pueden utilizar, sin embargo, todas ellas deben respetar el principio de la aleatoriedad.

En el presente artículo se desarrolla un diseño metodológico para la selección de una muestra representativa de estudiantes de una institución de educación superior, comenzando en la sección 2 con un marco conceptual de la teoría de muestreo, así como una contextualización de la misma. Posteriormente se propone una metodología para la estratificación, afijación, selección y estimación de una muestra de estudiantes universitarios finalizando con una discusión de la misma.

\section{DISEÑO MUESTRAL}

El muestreo probabilístico es una técnica metodológica que permite inferir características de una población a partir de la selección y posterior análisis de un conjunto de elementos pertenecientes a dicha población. Para ello es necesario elaborar un diseño muestral, el cual puede entenderse como una serie de especificaciones que detallan la metodología y los procesos empleados para la selección de una muestra probabilística a partir de una población dada.

Revista Economía y Administración (E\&A)

En la tabla 1 se presenta un breve resumen de los conceptos básicos de la teoría de muestreo, los cuales son necesarios para la comprensión del diseño metodológico que se propone en los apartados 2.2 al 2.6. 


\section{Tabla 1. Conceptos básicos de la teoría de muestreo}

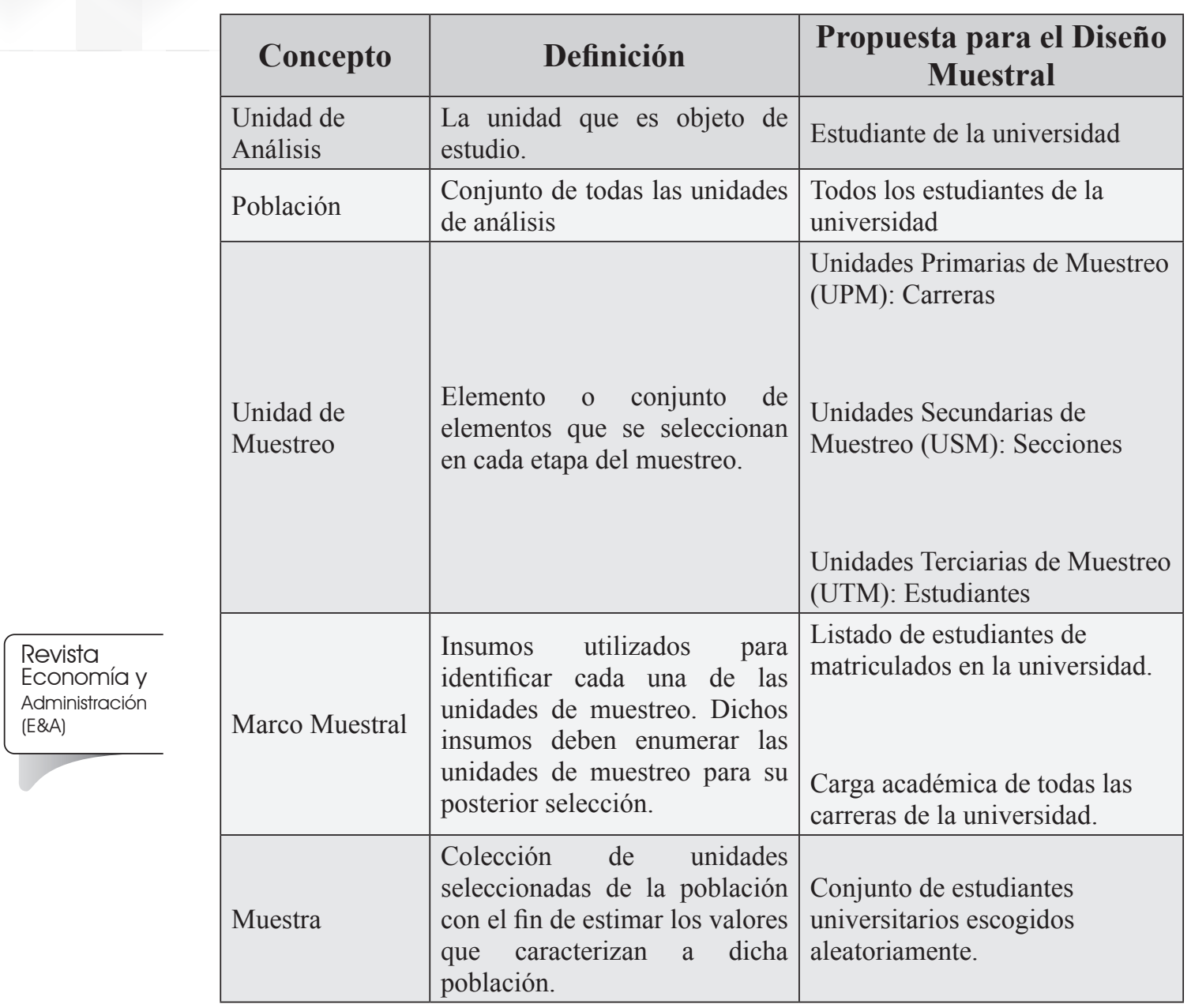

Fuente: Elaboración propia con base en (Vivanco, 2005). 


\subsection{TAMAÑO DE MUESTRA}

Una de las preguntas más comunes en la investigación social es referente al tamaño que debe tener una muestra para que los resultados obtenidos a partir de ella sean confiables, sin embargo, no existe una respuesta que sea universalmente correcta a esta interrogante. Elegir un tamaño adecuado para una muestra es un problema que se puede tornar muy complejo, por ello es necesario que se tenga claridad acerca del alcance de la investigación y del recurso con el que se dispone, asimismo se deben considerar los siguientes factores: a) Medida estadística sobre la cual se focalizará el análisis (proporciones, medias aritméticas, totales, etc.); b) Nivel del análisis (a nivel global, en subgrupos); c) Magnitud del error que se tolerará en las estimaciones calculadas a partir de la muestra; d) Nivel de confianza (probabilidad que las estimaciones tengan el error muestral deseado); e) Tamaño de la población (Briones, 1996).

En la práctica es muy complicado elaborar el diseño de una muestra y, específicamente definir su tamaño óptimo, para ello es necesario tener un conocimiento profundo de la población estudiada (Bustamante, 1998) $\mathrm{y}$, hacer una minuciosa revisión de la literatura que se ha escrito en investigaciones similares al tema central de estudio (Hernández, 2010), particularmente es conveniente tener una idea (sustentada teóricamente) de la dispersión de los elementos de la población con respecto a la(s)

Revista Economía y Administración (E\&A) variable(s) principal(es) del estudio, ya que para poblaciones más homogéneas normalmente se requieren muestras más pequeñas.

Sin embargo, si se desea elaborar una muestra con el fin de estimar proporciones y obtener conclusiones únicamente a un nivel global, es decir, no se quiere realizar inferencias en un subgrupo de la población, según (Cochran, 1977, págs. 75-76) es posible calcular el tamaño de la muestra por la fórmula:

Con $\mathrm{n}=\frac{\mathrm{n}_{0}}{1+\frac{\mathrm{n}_{0}}{\mathrm{~N}}}$

Donde, $\mathrm{n}_{0}=\frac{\mathrm{Z}^{2} \mathrm{p}(1-\mathrm{p})}{\mathrm{e}^{2}}$ 
$Z$ : Valor en la distribución normal estándar que garantiza un nivel de confianza prefijado.

$\mathrm{N}$ : Tamaño del marco muestral

P : Estimación de la proporción poblacional

E : Margen de error

Para minimizar el error en la selección del tamaño de la muestra, en caso de no conocer una estimación de, se debe utilizar . De esta forma, el tamaño de muestra necesario para estimar una proporción, con un nivel de confianza del $95 \%$ () y un margen de error de $5 \%$ es aproximadamente de ${ }^{1}$ :

Luego,

$$
\begin{gathered}
\mathrm{n}_{0}=\frac{(1.96)^{2}(0.5)(0.5)}{(0.05)^{2}}=384.16 \approx 385 \\
\mathrm{n}=\frac{385}{1+\frac{385}{\mathrm{~N}}}=\frac{385 \cdot \mathrm{N}}{\mathrm{N}+385}
\end{gathered}
$$

Revista Economía y Administración (E\&A)
Por lo tanto, sin importar el número de estudiantes matriculados en la universidad, el tamaño de la muestra necesaria para lograr estimaciones con un nivel de confianza del $95 \%$ y un error del $5 \%$ es a lo sumo de estudiantes, evidentemente cuando es relativamente pequeño, el valor de puede disminuir.

\subsection{ESTRATIFICACIÓN DE LA MUESTRA}

Después de calcular el tamaño de la muestra se procede a seleccionar estudiantes del marco muestral, sin embargo, el listado de todos los estudiantes matriculados en la universidad suele ser de carácter confidencial y no es posible tener acceso a él para investigaciones particulares, ya que posee información de contacto de los estudiantes. Una solución a este problema consiste en estratificar los estudiantes por facultades, carreras y secciones en las que se encuentran matriculados, para ello será necesaria la siguiente información:

- Número de estudiantes adscritos a cada carrera impartida en la universidad en el periodo académico en el que se realiza la investigación, que a partir de ahora será denotado como periodo t.

1 Un error común es asumir que el nivel de confianza y el margen de error deben sumar 100\%, lo cual es falso. El margen de error es una cota superior de la diferencia absoluta que se predice teóricamente entre el parámetro poblacional y la estadistica calculada a partir de la muestra. El nivel de confianza mide la probabilidad que una muestra dada arroje una estadística dentro del margen de error previamente establecido. 
- Carga académica de la universidad, es decir, un listado de todas las asignaturas y secciones que se imparten (en el periodo t) con la cantidad de estudiantes que éstas poseen.

Nota: La información requerida no contempla nombres ni información de contacto de estudiantes, solamente la oferta académica de la universidad y la cantidad de estudiantes matriculados por carrera y secciones en el periodo académico t, por lo que esta información suele ser de dominio público en las instituciones de educación superior.

La estratificación del marco muestral consiste en una agrupación de estudiantes por medio de la facultad a la que se encuentran adscritos, posteriormente se identifican todas las carreras contenidas en cada una de las facultades y finalmente se toman todas las secciones ${ }^{2}$ de todas las asignaturas pertenecientes al plan de estudios de cada carrera (véase Figura 1).

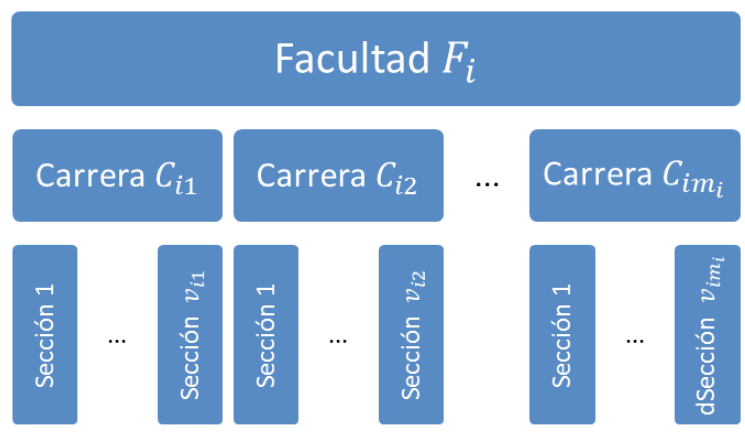

Revista

Economía y Administración (E\&A)

Figura 1. Estratificación del marco muestral

Fuente: Elaboración propia

\subsection{AFIJACIÓN DE LA MUESTRA}

La distribución de la muestra en los distintos estratos será proporcional al número de estudiantes que pertenezcan a dichos estratos. Para ello será necesario definir la siguiente nomenclatura:

$\mathrm{N}_{\mathrm{i}}$ : Número de estudiantes adscritos a la facultad $\mathrm{Fi}$

$\mathrm{N}_{\mathrm{ij}}$ : Número de estudiantes adscritos a la carrera $\mathrm{Cij}$

$\mathrm{n}_{\mathrm{i}}$ Tamaño de la muestra que le corresponde a la facultad $\mathrm{Fi}$

2 Las secciones deben ser todas las que se estén impartiendo en el periodo t. 
De esta forma, se seleccionarán $\mathrm{n}_{\mathrm{i}}=\frac{\mathrm{N}_{\mathrm{i}}}{\mathrm{N}}$ estudiantes adscritos a la facultad para conformar la muestra que será de tamaño $n=\sum n_{i}$.

\subsection{SELECCIÓN DE LA MUESTRA}

El tipo de muestreo utilizado será estratificado, probabilístico y trietápico. La unidad primaria de muestreo (UPM) son las carreras, la unidad secundaria de muestreo (USM) son las secciones y la unidad terciaria de muestreo (UTM) son los estudiantes dentro de cada sección. El proceso de selección de la muestra se describe a continuación:

a. Selección de las UPM's

En esta etapa se seleccionará aleatoriamente una carrera $\mathrm{C}_{\mathrm{ij}}$ dentro de cada facultad $\mathrm{F}_{\mathrm{i}}$, cada carrera tendrá una probabilidad de ser elegida directamente proporcional a la cantidad de estudiantes que ésta tenga, asignándole un peso $\mathrm{w}_{\mathrm{ij}}$ a cada carrera $\mathrm{C}_{\mathrm{ij}}$ mediante la fórmula:

Revista Economía y Administración (E\&A)

$$
w_{i j}=\frac{N_{i j}}{N i}
$$

b. Selección de las USM's

Una vez seleccionada una carrera $C_{i j}$ de cada facultad $F_{i}$, se procede a seleccionar aleatoriamente una sección de cualquier asignatura contemplada en el plan de estudios de la carrera $C_{i j}$. Para ello se le asignará a cada sección de $\mathrm{C}_{\mathrm{ij}}$ un peso bajo los siguientes criterios:

i. Si la sección pertenece a una asignatura exclusiva de la carrera $\mathrm{C}_{\mathrm{ij}}$, el peso de dicha sección será su número de estudiantes.

ii. Si la sección pertenece a una asignatura que está incluida en el plan de estudios de carreras: $\mathrm{C}_{\mathrm{ij}}, \mathrm{C}_{\mathrm{a}_{2} \mathrm{~b}_{2}}, \cdots \mathrm{C}_{\mathrm{a}_{\mathrm{m}} \mathrm{b}_{\mathrm{m}}}$, el peso de la sección será:

Donde, $w=\frac{N_{i j}}{N_{i j}+\sum_{l=2}^{m} N_{a_{1} b_{1}}} k$

$N_{i j}$ : Número de estudiantes de la carrera

$N_{\text {aibi }}$ : Número de estudiantes matriculados en la carrera

$k$ : Número de estudiantes de la sección 
Nota: El proceso b.i. garantiza que las secciones exclusivas de la carrera $C i j$ tengan un peso proporcional al número de estudiantes que poseen. Mientras que el proceso b.ii. garantiza que las secciones que pertenecen a más de una carrera estén representadas por un peso teórico, manteniendo una proporción del número de estudiantes de la carrara $C i j$ en relación al número de estudiantes de todas las carreras que contemplan esa asignatura en sus planes de estudios.

\section{c. Selección de las UTM's}

En esta etapa se tiene seleccionada la carrera $C_{i j}$ y una sección perteneciente a dicha carrera. Ahora se procede a seleccionar la muestra de tamaño $n_{i}$ que representará a toda la facultad $F_{i}$ bajo los siguientes criterios:

Sea $k$ el número de estudiantes de la sección y $r$ el número de estudiantes de la sección que pertenecen a la carrera $C_{i j}$.

i. Si $r \leq n_{i}$ : los estudiantes pasan a formar parte de la muestra.

ii. Si $n_{i}<r$ : Se realizará un muestreo aleatorio simple de los estudiantes de la sección para obtener una muestra de tamaño $n_{i}$.

Si ocurre el proceso c.i. será necesario regresar al proceso b. y seleccionar otra sección de la carrera $C_{i j}$ de forma aleatoria, teniendo en cuenta que la(s) sección(es) escogidas previamente no pueden ser elegibles, es decir, se elabora un muestreo sin remplazo. Una vez escogida una nueva sección se procede con el proceso c. sustituyendo el valor de $n_{i}$ por $s=n_{i}-r$ (la cantidad de estudiantes que faltan para completar la muestra de $F_{i}$ )

\subsection{ESTIMACIÓN}

El proceso de selección de la muestra es completamente aleatorio, por lo que es posible calcular la probabilidad que tiene un estudiante de la facultad de haber sido escogido en una muestra y se puede calcular de la siguiente forma:

La probabilidad de seleccionar una carrera $C_{i j}$ de la facultad $F_{i}$ es:

$P_{i j 1}=\frac{N_{i j}}{N i}$ 
La probabilidad de seleccionar una sección $v$ de la carrera $C_{i j}$ es:

Donde $^{3}, P_{i j 2}=\frac{\mathrm{w}_{\mathrm{v}}}{\sum_{\mathrm{l}=1}^{\mathrm{v}_{\mathrm{ij}}} \mathrm{w}_{\mathrm{l}}}$

Wv : Peso de la sección de la carrera .

$\mathrm{V}_{\mathrm{ij}}$ : Número de secciones pertenecientes a la carrera .

$\mathrm{W}_{\mathrm{I}}$ : Peso de la sección de la carrera $C_{i j}$.

La probabilidad de seleccionar un estudiante de una sección es:

Por tanto, la probabilidad de escoger un estudiante de una sección de la carrera de la facultad de una institución de educación superior es de y su respectivo factor de expansión será de .

\section{DISCUSIÓN Y CONCLUSIONES}

Elaborar una muestra representativa de una población es un tema muy complejo que requiere de mucho análisis en todas sus instancias, así como una reflexión

Revista Economía y Administración (E\&A) profunda del fenómeno que se desea estudiar. También es necesario elaborar una extensa revisión bibliográfica de investigaciones realizadas por otros autores en el mismo tema, dado que información adicional como una medida de la homogeneidad de la población con respecto a la(s) variable(s) de estudio o una estimación de los parámetros poblacionales en contextos similares podrían ser de mucha utilidad para calcular tamaños de muestras más precisos y confiables para la investigación, lo que podría conducir a reducir costos a través de una reducción en la muestra, manteniendo siempre altos estándares de confianza y márgenes de errores relativamente pequeños.

Una característica que siempre debe estar presente en un diseño muestral es la aleatoriedad ya que, para alcanzar la representatividad de una muestra, se debe garantizar que todos las unidades de análisis del marco muestral tengan teóricamente la misma probabilidad de ser escogidas en la muestra, o al menos una probabilidad conocida para cada elemento. De esta forma se evita la adhesión (voluntaria o involuntaria) de sesgo a los resultados, dado que el investigador es indiferente a la elección de las unidades de muestreo. Entonces, bajo esta premisa de aleatoriedad y con un tamaño de muestra apropiado, teóricamente se garantiza (por el teorema del límite central y la ley de los grandes números, véase (Vivanco, 2005, pág. 25)) que las características más comunes en la población se verán plasmadas en la muestra, logrando la representatividad de la misma. 
El diseño muestral presentado en este artículo, puede ser útil al lector para seleccionar una muestra de estudiantes universitarios si desea estudiar algunas características generales en toda la población, sin distinguir entre otras variables como edad, sexo, jornada de estudio, nivel socioeconómico, etc. Una forma de contemplar estas variables en la investigación, bajo este mismo diseño muestral, es tomando muestras proporcionales e independientes en cada dominio, es decir, si se desea contemplar la variable sexo, se debe seleccionar dos muestras bajo esta misma metodología, una muestra de hombres y una de mujeres manteniendo la misma proporción en la muestra y en la población, es decir, si el $60 \%$ de los estudiantes universitarios son hombres, el $60 \%$ de los estudiantes de la muestra deben ser hombres. Evidentemente el tamaño de la muestra aumentará a medida que se busque la representatividad en más subgrupos de la población. Para muestras más complejas será necesario utilizar otros tipos de muestreo o una combinación de ellos, como el muestreo por conglomerados, o el muestreo sistemático, que quizá logre mantener los estándares de confianza disminuyendo considerablemente el tamaño de la muestra. 


\section{BIBLIOGRAFÍA}

Arana, J. M., Blanco, C., Meilán, J. J., Pérez, E., Carro, J., \& Gordillo, F. (2011). The impact of poly drug use on several prospective memory measures in a sample of university students. Revista Latinoamericana de Psicología, 43(2), 229-240.

Arué, R. L. (2011). Trabajo, estudiantes y graduados universitarios. Una relación temprana y conflictiva. Educación, Lenguaje y Sociedad, 8(8), 151-169.

Avendaño, C. S. (2005). Los Problemas de Redacción de los Estudiantes Costarricenses: Una Propuesta de Revisión desde la Lingüística del Texto. Revista de Filología y Linguística de la Universidad de Costa Rica, 31(1), 267-295.

Briones, G. (1996). Metodología de la Investigación Cuantitativa en las Ciencias Sociales. Bogotá, Colombia: ICFES.

Revista Economía y Administración (E\&A)
Bustamante, F. (1998). Teoría del Muestreo: Particularidades del Diseño Muestral en Estudios de la Conducta Social. Revista Electrónica de Metodología Aplicada, 3(1), 1-15.

Castro-González, K. C., Delgado-Ortiz, C., \& Rodríguez-Madera, J. (2013). Uso y Manejo del Crédito en Estudiantes Universitarios., 8, págs. 1344-1349.

Cochran, W. (1977). Sampling Techniques. Harvard University: John Wiley \& Sons.

Echavarría Grajales, C. V., Buítrago, A. O., \& Álvarez Rincón, Á. A. (2008). Perspectiva ético-política del ser ciudadano: una mirada desde los jóvenes. Desafíos, 18, 182-212.

Galindo Ramírez, L., Cubides Martínez, J., \& Acosta Sánchez, F. (2010). Política y juventud en la vida universitaria:un estudio de caso. Utopia y Praxis Latinoamericana, 15(50), 71-90.

González Ramírez, M. T., Hernández, R. L., \& García-Campayo, J. (2009). Relación entre la depresión, la ansiedad y los síntomas psicosomáticos en una muestra de estudiantes universitarios del norte de México. Pan American Journal of Public Health, 25(2), 141-145. 
Hernández Sampieri, R., Fernández Collado, C., \& Baptista Lucio, P. (2010). Metodología de la investigación. McGraw-Hill.

Inzunsa Cazares, S., \& Jiménez Ramírez, J. V. (2013). Caracterización del Razonamiento Estadístico de Estudiantes Universitarios Acerca de las Pruebas de Hipótesis. Revista Latinoamericana de Investigación en Matemática Educativa, 16(2), 179-211.

Miratía Moncada, O. (2010). Efectos de la web y las TIC en el desempeño y rendimiento de estudiantes universitarios de computación en modalidad a distancia. Revista de Pedagogía, 31(88), 97-131.

Núñez, J., Miranda, L., \& Scavia, J. (2009). Estudios de Economía y Cooperación Social. Trimestre Económico, 76(3), 695-720.

Ortega, C., \& Eguía, I. (2011). Demanda y oefrta de educación superior: Integración total de la cadena de valor y las cadenas de suministro. Economía y administración, 2(1), 21-50.

Pérez, B. J. (2007). Competitividad, desarrollo e ingeniería: algunas definiciones y reflexiones. Ingeniería y Competitividad, 9(1), 57-75.

Vásquez, E. M., Cunningham, J., Brands, B., Strike, C., \& da Gloria Miotto Wright, M. (2009). Consumo Percibido y Uso de Drogas Lícitas e Ilícitas

Revista Economía y Administración (E\&A) en Estudiantes Universitarios en la Ciudad de Medellín, Colombia. Revista Latino-Americana de Enfermagem, 17(3), 886-892.

Vivanco, M. (2005). Muestreo Estadistico. Diseño Y Aplicaciones. Santiago de Chile: Editorial Universitaria.

Este articulo fue seleccionado de trabajos presentados en la Primera Conferencia Internacional sobre Economía, Administración y Tecnología, evento organizado por el Consorcio Economía, Administración y Tecnología (CEAT). El documento original ha seguido el proceso de revisión estándar de la Revista Economía y Administración (E\&A). El proceso fue dirigido por Marvin Aguilar (CEAT-2013) y supervisado por el PhD. Jorge Flores Silva, MSc. Manuel Flores Fonseca y PhD. Jesús Argueta Moreno (Editores de E\&A). 